Mitsue Isosaki

\title{
Absenteísmo entre Trabalhadores de Serviços de Nutrição e Dietética de Dois Hospitais em São Paulo
}

Diretora técnica do Serviço de Nutrição e Dietética do Instituto do Coração do Hospital das Clínicas da Faculdade de Medicina da Universidade de São Paulo, mestre em Saúde Pública pelo Departamento de Saúde Ambiental da Faculdade de Saúde Pública da USP

Agradecimentos

Aos servidores e à diretoria dos hospitais participantes, pelo apoio e colaboração; ao Prof. Emérito Diogo Pupo Nogueira pela orientação e ao Professor Titular José Maria Pacheco e à doutoranda Nívea Matuda, pela contribuição nas análises estatísticas.

\section{Absenteeism among Dieticians and Nutrition in Two Hospitals in São Paulo}

Estudo retrospectivo, com participação de 285 servidores de Serviços de Nutrição e Dietética de uma instituição de excelência pública e uma privada. Para a quantificação das ausências foram utilizadas informações coletadas nas escalas mensais dos trabalhadores referentes ao período de janeiro a dezembro de 2001, formulações baseadas nas recomendações da Subcomissão de Absenteísmo da Comissão Permanente e Associação Internacional de Medicina do Trabalho e análises fornecidas pelos Serviços de Medicina do Trabalho. Foi aplicado um questionário de auto-avaliação das condições de trabalho e de opinião dos servidores sobre a questão do absenteísmo. O absenteísmo de trabalhadores nos Serviços de Nutrição e Dietética se assemelhou entre os hospitais público e privado tanto quanto às outras organizações não hospitalares, o que direciona em agravante por se tratar de instituições de saúde onde o figurante central é o doente, mas que os próprios trabalhadores requerem uma atenção na melhoria da qualidade de vida no trabalho.

Palavras-chaves Absenteísmo, Serviço de Nutrição e Dietética, Hospital, Condições de trabalho, Trabalhadores.

Retrospective study consisted of 285 workers of a public and a private hospital. In order to obtain data on absences information was collected on workers monthly scales regarding the period from January to December 2001, and statements were based on the recommendations of the Absenteeism Sub-Committee of the Permanent Health and analyses were given by the Services of Occupational Health. A self-administered questionnaire about conditions of work and workers opinion abouth the issue was used. The absenteeism of Dieticians and Nutrition workers did not differ as between the public and the private hospital as from that of workers in other sectors. Thus the question is of great importance because, although the patient is the person most affected, the employee also calls for attention regarding an improvement of his working conditions.

Keywords Absenteeism, Hospital Food Service, Hospital, Working Conditions, Workers. 


\section{Introdução}

O absenteísmo é definido como ausência do servidor ao trabalho, por qualquer razão, naquelas ocasiões em que seria de esperar a sua presença. Estudos sobre a questão datam de muito tempo e essas pesquisas referem que não existem causas específicas para o absenteísmo dos trabalhadores e que o mesmo decorre de uma soma de fatores (demográficos, biológicos, sociais, de organização do trabalho, psicossociais) que tornam o problema de difícil solução, principalmente, por envolver a questão comportamental (NOGUEIRA, 1980).

Na área específica de Serviços de Alimentação e de Nutrição e Dietética são raros os estudos em relação aos demais setores e áreas. Os Serviços de Nutrição e Dietética (SND) têm como característica a prestação diária, ininterrupta e contínua de serviço, sob rigorosos padrões de qualidade, independente do número de funcionários presentes no dia. Assim, se a falta de um elemento da equipe gera atrasos e sobrecarrega os trabalhadores presentes, a situação torna-se crítica se houver várias ausências no dia e/ou se essas ausências não forem momentâneas. A questão do absenteísmo nesses serviços é um dos principais problemas que afeta não só a eficácia, mas principalmente o atendimento prestado aos pacientes, tanto em instituições públicas quanto privadas. A partir da pergunta de partida dos motivos e das características do absenteísmo entre trabalhadores de Serviços de Nutrição e Dietética dos hospitais e partindo do pressuposto de que há diferentes motivos entre um hospital público e privado, o estudo teve como objetivo geral caracterizar o absenteísmo de trabalhadores em dois Serviços de Nutrição e Dietética hospitalar, um público e outro privado, sediados na cidade de São Paulo.

\section{Métodos}

A pesquisa foi realizada em dois hospitais considerados de excelência em seus campos de atuação, 1 público, denominado $A$, com 427 leitos, sendo cerca de $75 \%$ destinados ao atendimento de pacientes SUS, com cerca de 2.900 servidores, sendo em torno de $4 \%$ pertencentes ao SND. O outro hospital privado, denominado B, com cerca de 400 leitos sendo $8 \%$ destinados aos pacientes não pagantes e com cerca de 2.300 funcionários, sendo $7 \%$ pertencentes ao SND.

A população total do estudo constou de 123 servidores do SND do hospital A e de 162 do SND do hospital B de todas as categorias funcionais que estavam na ativa no ano de 2001. Não foram incluídos no estudo os responsáveis pelo SND, os servidores com menos de 3 meses de contratação e os servidores que estiveram afastados durante o ano de 2001.

\section{Coleta de dados}

As ausências de cada servidor participante do estudo foram coletadas a partir das ocorrências registradas em escalas mensais dos SNDs dos dois hospitais, referentes ao período de janeiro a dezembro de 2001.

As causas das ausências por doenças e por acidentes de trabalho foram obtidas junto aos Serviços de Medicina do Trabalho dos dois hospitais.

Para verificar a opinião dos trabalhadores sobre as condições de trabalho (FISCHER, 1990) e a questão do absenteísmo foram utilizados questionários de auto-avaliação, aplicados uma única vez, elaborados especialmente para esse fim e previamente testados. A participação na pesquisa foi voluntária e aqueles que concordaram assinaram o termo de consentimento esclarecido, conforme regimentos das Comissões de Ética em Pesquisa das instituições envolvidas, bem como de acordo com a Resolução 196/96 do Conselho Nacional de Saúde.

\section{Tratamento dos dados}

Para a quantificação das ausências dos trabalhadores foram utilizados indicadores recomendados pela Subcomissão de Absenteísmo da Comissão Permanente e Associação Internacional de Medicina do Trabalho (MONIZ, 1978; NOGUEIRA, 1980).

Para o cálculo das taxas não foram computadas as ausências por motivos legais constantes na Consolidação das Leis do Trabalho (CLT) e na Constituição da República Federativa do Brasil, bem como as dos servidores em auxílio doença pelo Instituto Nacional do Seguro Social (I.N.S.S.) há mais de 1 ano.

A duração das ausências por doença própria foi dividida em de curta duração ( 1 a 3 
dias), de longa duração (4 a 15 dias) e licença I.N.S.S. (maior que 15 dias) (MONIZ, 1978).

Para o cálculo do tempo de exposição (população em risco) multiplicou-se o número de empregados pelo número de dias do ano (365 dias descontados os 30 dias de férias) e subtraiu-se o número total de dias de ausências (exceto o primeiro dia do episódio, devido o trabalhador estar sob risco de faltar ao trabalho nesse dia) e o número total de dias não trabalhados dos servidores admitidos e demitidos no ano.

Os motivos das ausências foram quantificados por número de episódios e classificados em: doença própria (categorizada em licença médica de curta duração, de longa duração e licença I.N.S.S.), doença na família (afastamento do servidor por doença do filho), acidente de trabalho, legal (licença maternidade, paternidade, nojo, gala, doação de sangue e outros constantes na CLT e na Constituição da República Federativa do Brasil), voluntário (faltas abonadas, justificadas e injustificadas) e compulsório (ausências por aplicação de pena disciplinar por parte do empregador).

As causas das licenças médicas por doenças foram agrupadas por especialidade, devido a não obtenção de dados segundo o Código Internacional de Doenças (CID) no Hospital B.

Os acidentes de trabalho foram analisados por local de ocorrência, dia da semana, horário de ocorrência após início do trabalho, causas do acidente, conseqüência do acidente e parte do corpo atingida.

Para as análises estatísticas foram utilizados:

- Teste de hipótese de homogeneidade de proporção (teste exato de Fisher quando as freqüências eram baixas e Teste de quiquadrado de Pearson ) para verificar se havia diferença entre os funcionários dos hospitais A e B;

- Modelos loglineares de binomial negativa para verificação da significância das razões das taxas de freqüência por episódios e das razões de proporção de dias perdidos, escolhidos por representarem, dentre todos os indicadores, uma taxa genuína de incidência e de dias efetivamente perdidos pelos trabalhadores;

- Modelos lineares a fim de averiguar a influência do tipo de hospital e do hábito de faltar sobre a opinião dos funcionários e

- Análises descritivas em tabelas com falta de independência entre os eventos considerados (episódios ou casos de doença de um mesmo funcionário e respostas múltiplas).

Os níveis de significância estabelecidos para as análises foram de $10 \%$ e de $20 \%$.

Para as análises foram utilizados os Programas estatísticos SAS, S-PLUS e STATA.

\section{Resultados}

A caracterização da população do estudo está descrita na TABELA 1. Em ambos os hospitais, a grande maioria era constituída de mulheres.

O cálculo dos indicadores revelou taxas de freqüência por indivíduo e por episódio e proporção de tempo perdido mais elevadas no hospital $A$ em relação ao $B$ em 14\%, 15\% e 3\%, respectivamente. Já a duração média dos episódios e a média de dias perdidos por trabalhador faltante foram inferiores no hospital $A$ em relação ao $B$ em $12 \%$. A taxa de duração e a média de episódios por trabalhador faltante foram semelhantes. 
Tabela 1 Distribuição dos participantes dos Hospitais A e B, segundo características dos trabalhadores, São Paulo, 2001.

\begin{tabular}{|c|c|c|c|c|c|c|}
\hline \multirow[t]{3}{*}{ Característica } & \multirow[t]{3}{*}{ Categoria } & \multicolumn{4}{|c|}{ Hospital } & \multirow{3}{*}{$\mathrm{p}^{*}$} \\
\hline & & \multicolumn{2}{|c|}{$\underset{n=123}{A}$} & \multicolumn{2}{|c|}{$\underset{n=162}{B}$} & \\
\hline & & $\mathrm{N}^{\mathrm{o}}$ & $\%$ & $\mathrm{~N}^{\mathrm{o}}$ & $\%$ & \\
\hline \multirow[t]{2}{*}{ Gênero } & Homem & 17 & 14 & 53 & 33 & $0,0002^{(a)}$ \\
\hline & Mulher & 106 & 86 & 109 & 67 & \\
\hline \multirow{2}{*}{$\begin{array}{l}\text { Faixa etária } \\
\text { (anos) }\end{array}$} & 20 a 39 & 63 & 51 & 122 & 75 & $<0,0001^{(\mathrm{a})}$ \\
\hline & $40 e+$ & 60 & 49 & 40 & 25 & \\
\hline \multirow[t]{3}{*}{ Estado conjugal } & Solteiro & 54 & 44 & 42 & 26 & $0,0025^{(a)}$ \\
\hline & $\begin{array}{l}\text { Casado/vive com } \\
\text { companheiro }\end{array}$ & 53 & 43 & 102 & 63 & \\
\hline & Separado/viúvo & 16 & 13 & 18 & 11 & \\
\hline \multirow{3}{*}{$\begin{array}{l}\text { Tempo de trabalho } \\
\text { no Serviço } \\
\text { (anos) }\end{array}$} & 0 a 4 & 53 & 43 & 82 & 50 & $0,0007^{(a)}$ \\
\hline & 5 a 14 & 39 & 32 & 66 & 41 & \\
\hline & $15 e+$ & 31 & 25 & 14 & 9 & \\
\hline \multirow[t]{4}{*}{ Área de atuação } & Produção & 59 & 48 & 82 & 51 & $0,2766^{(b)}$ \\
\hline & Internação & 58 & 48 & 77 & 47 & \\
\hline & Ambulatório & 3 & 2 & 0 & 0 & \\
\hline & Expediente & 3 & 2 & 3 & 2 & \\
\hline \multirow{2}{*}{$\begin{array}{l}\text { Responsabilidade } \\
\text { com crianças }\end{array}$} & Sim & 60 & 49 & 93 & 57 & $0,1480^{(a)}$ \\
\hline & Não & 63 & 51 & 69 & 43 & \\
\hline
\end{tabular}

(a) Teste de homogeneidade de proporção (qui-quadrado de Pearson).

(b) Teste exato de Fisher (bilateral).

* Probabilidade de rejeitar a hipótese de homogeneidade quando verdadeira.

Na análise estatística dos indicadores, comparando as taxas de freqüência por episódios ( TABELA 2) dos hospitais A e B, verificou-se que a razão estimada foi de 1,16, mas não significativa. Entre homens e mulheres, a razão foi significativa somente para os funcionários que tinham sob seus cuidados crianças e estimada em 0,48 , ou seja entre os homens com responsabilidade por crianças, nos dois hospitais, a taxa foi $52 \%$ menor em relação às mulheres.

Em relação ao tempo de trabalho houve diferença significativa somente entre funcionários com 5 a 14 anos e 15 ou mais de trabalho, sendo a taxa $56 \%$ maior.

O fato de ter responsabilidade com crianças foi significativo somente entre as mulheres, em $116 \%$ entre aquelas com responsabilidade em relação as que não tinham esta responsabilidade. 
Tabela 2 Razão estimada e intervalo de confiança das taxas de freqüência por episódios dos Hospitais A e B, São Paulo, 2001.

\begin{tabular}{|c|c|c|c|}
\hline Variável & Razão & Estimativa & $\begin{array}{c}\text { Intervalo de } \\
\text { confiança } 95 \%\end{array}$ \\
\hline Hospital & $A / B$ & 1,16 & $0,88-1,53$ \\
\hline \multirow[t]{2}{*}{ Gênero } & $\begin{array}{l}\text { Homem/Mulher } \\
\text { (com responsabilidade } \\
\text { com crianças) }\end{array}$ & 0,48 & $0,30-0,76$ \\
\hline & $\begin{array}{l}\text { Homem/Mulher } \\
\text { (sem responsabilidade } \\
\text { com crianças) }\end{array}$ & 1,00 & $0,63-1,57$ \\
\hline \multirow[t]{3}{*}{ Tempo de trabalho } & 0-4 / 5-14 anos & 0,94 & $0,71-1,26$ \\
\hline & $0-4 / 15 e+$ anos & 1,47 & $0,98-2,19$ \\
\hline & $5-14 / 15$ e + anos & 1,56 & $1,03-2,35$ \\
\hline \multirow{2}{*}{$\begin{array}{l}\text { Responsabilidade } \\
\text { com crianças }\end{array}$} & Sim/Não (homem) & 1,03 & $0,58-1,81$ \\
\hline & Sim/Não (mulher) & 2,16 & $1,58-2,95$ \\
\hline \multicolumn{2}{|c|}{$\begin{array}{l}\text { Na análise comparativa das proporções de } \\
\text { dias perdidos ( TABELA 3) dos hospitais A e } \\
\text { B foram significativos os efeitos de gênero } \\
\text { (os dias perdidos entre homens foi } 50 \% \text { me- } \\
\text { nor em relação às mulheres, nos dois hospi- } \\
\text { tais), tempo de trabalho ( } 91 \% \text { e } 100 \% \text { maior }\end{array}$} & \multicolumn{2}{|c|}{$\begin{array}{l}\text { entre servidores com } 0 \text { a } 4 \text { anos e } 5 \text { a } 14 \\
\text { anos, respectivamente, em relação a } 15 \text { anos } \\
\text { e mais) e responsabilidade com crianças (62\% } \\
\text { maior entre os trabalhadores com responsabi- } \\
\text { idade comparado com os que não tinham essa } \\
\text { responsabilidade em ambos os hospitais). }\end{array}$} \\
\hline
\end{tabular}

Tabela 3 Razão estimada e intervalo de confiança da proporção de dias perdidos dos Hospitais A e B, São Paulo, 2001.

\begin{tabular}{llcc}
\hline Variável & Razão & Estimativa & $\begin{array}{c}\text { Intervalo de } \\
\text { confiança } 95 \%\end{array}$ \\
\hline Hospital & A/B & 1,01 & $0,68-1,49$ \\
Gênero & Homem/Mulher & 0,50 & $0,31-0,79$ \\
Tempo de trabalho & $0-4 / 5-14$ anos & 0,95 & $0,64-1,42$ \\
& $0-4 / 15 e+$ anos & 1,91 & $1,11-3,27$ \\
Responsabilidade & $5-14 / 15$ e + anos & 2,00 & $1,16-3,47$ \\
Com criança & Sim / Não & 1,62 & $1,12-2,36$ \\
& & & \\
\hline
\end{tabular}




\section{Motivos das ausências}

Em ambos os hospitais, os episódios de doença própria (TABELA 4) contribuíram com o maior percentual, seguidos por motivos voluntários com percentual bem maior no hospital A que no hospital B. As ausências por doença própria de curta duração representaram $75 \%$ no hospital A e $73 \%$ no hospital $B$.

As doenças do sistema osteomuscular e do tecido conjuntivo foram as mais freqüentes em ambos os hospitais (19\% no hospital A e $32 \%$ no hospital B), seguidas das doenças do aparelho digestivo (17\%) no hospital $\mathrm{Ae}$ do aparelho respiratório (16\%) no hospital B.

Quanto aos acidentes de trabalho ocorreram em maior proporção, em ambos os locais, no próprio serviço (54\% no hospital A e $63 \%$ no hospital B) e no primeiro período da jornada de trabalho. As instalações (piso, vapor) e os equipamentos específicos da área, incluindo utensílios como facas e carros para transporte dos alimentos foram os maiores responsáveis pelos acidentes $(50 \%$ no hospital A decorrente de instalações e $46 \%$ no hospital $B$ devido aos equipamentos), os quais provocaram contusão em $58 \%$ dos trabalhadores no hospital A e ferimentos com corte em $54 \%$ dos trabalhadores do hospital B e afetaram em maior proporção os membros superiores $159 \%$ no hospital A e $71 \%$ no hospital B).
Da segunda fase da pesquisa ou seja de coleta de opiniões dos trabalhadores sobre as condições de trabalho e a questão do absenteísmo participaram 112 servidores do hospital A e 140 do hospital B, correspondendo a $91 \%$ e $86 \%$,respectivamente, dos participantes da fase I. Dentre esses, os absenteístas foram em maior número.

Quanto à opinião dos participantes sobre os motivos do absenteísmo daqueles que costumam faltar ao serviço, no hospital B, em ambos os grupos, absenteístas e não absenteístas, referiram "doença própria". Já entre os trabalhadores do hospital A, o motivo "de ordem pessoal" foi o mais citado entre os absenteístas e os motivos "de ordem pessoal, familiar, doença própria e a insatisfação no trabalho" entre os não absenteístas.

Os trabalhadores quando questionados sobre os motivos da assiduidade daqueles que nunca faltam ao trabalho, a resposta mais citada entre os absenteístas do hospital A foi "gostam do que fazem" e entre os não absenteístas "preocupação com o serviço". Já no hospital B tanto os absenteístas quanto os não absenteístas responderam "preocupação com o serviço".

Foi unânime a opinião dos trabalhadores quanto ao prejuízo das ausências no desempenho do Serviço tanto entre absenteístas quanto não absenteístas de ambos os hospitais. Pela análise estatística verificou-se que somente o efeito de hospital foi significativo a

Tabela 4 Distribuição dos episódios de ausências dos Hospitais A e B por motivos, São Paulo, 2001.

\begin{tabular}{lrrrrrrrr}
\hline \multirow{2}{*}{ Motivo } & \multicolumn{3}{c}{ Hospital } & & & \\
\cline { 3 - 7 } & \multicolumn{2}{c}{$\mathrm{A}$} & & $\mathrm{B}$ & & \multicolumn{2}{c}{ Total } \\
& $\mathrm{N}^{\circ}$ & $\%$ & $\mathrm{~N}^{\circ}$ & $\%$ & $\mathrm{~N}^{\circ}$ & $\%$ \\
\hline Doença própria & 180 & 43 & 373 & 75 & 553 & 61 \\
Doença na família & 59 & 14 & 0 & 0 & 59 & 6 \\
Acidente trabalho & 24 & 6 & 24 & 5 & 48 & 5 \\
Voluntário & 145 & 34 & 82 & 17 & 227 & 25 \\
Legal & 8 & 2 & 14 & 3 & 22 & 2 \\
Compulsório & 4 & 1 & 1 & 0 & 5 & 1 \\
\hline Total & 420 & 100 & 494 & 100 & 914 & 100 \\
\hline
\end{tabular}


um alfa $=20 \%$ sobre a proporção dos que concordam com o prejuízo no desempenho do serviço e como esse efeito foi negativo, estimado em $4 \%$, proporcionalmente houve menos funcionários do hospital $B$ concordando com essa opinião comparados aos do hospital A.

As sugestões apresentadas pelos trabalha- dores para diminuição das ausências ao trabalho foram, tanto entre absenteístas quanto não absenteístas do hospital A: "melhorar as condições de trabalho". Do hospital B, os absenteístas sugeriram "absenteístas deveriam ser ouvidos pelas chefias" e os não absenteístas "maior rigor das chefias com os absenteístas".

Tabela 5 Escore médio estimado das avaliações de condições de trabalho, segundo itens pesquisados entre absenteístas e não absenteístas dos Hospitais A e B, São Paulo, 2001.

\begin{tabular}{|c|c|c|c|c|}
\hline \multirow[b]{2}{*}{ Item } & \multicolumn{2}{|c|}{ Absenteísta } & \multicolumn{2}{|c|}{ Não absenteísta } \\
\hline & $\begin{array}{c}\text { A } \\
n=87\end{array}$ & $\begin{array}{c}\mathrm{B} \\
\mathrm{n}=99\end{array}$ & $\begin{array}{c}\mathrm{A} \\
\mathrm{n}=25\end{array}$ & $\begin{array}{c}\text { B } \\
n=41\end{array}$ \\
\hline Risco acidente & 3,1 & 3,8 & 3,3 & 4,0 \\
\hline Ruído & 3,0 & 3,4 & 2,6 & 3,0 \\
\hline Calor & 2,6 & 2,6 & 2,6 & 2,6 \\
\hline Frio & 3,8 & 3,8 & 3,8 & 3,8 \\
\hline Iluminação & 3,6 & 4,7 & 3,6 & 4,7 \\
\hline Espaço trabalho & 2,1 & 4,2 & 2,1 & 4,2 \\
\hline Trab. Monótono & 3,4 & 3,7 & 3,8 & 4,0 \\
\hline $\begin{array}{l}\text { Trab.exige muito } \\
\text { esforço mental }\end{array}$ & 3,5 & 3,5 & 3,5 & 3,5 \\
\hline $\begin{array}{l}\text { Trab. exige muito } \\
\text { esforço físico }\end{array}$ & 2,5 & 3,2 & 3,1 & 3,8 \\
\hline Ritmo trabalho & 2,7 & 3,5 & 3,0 & 3,7 \\
\hline $\begin{array}{l}\text { Relacionamento } \\
\text { c/colegas trabalho }\end{array}$ & 3,1 & 3,5 & 3,1 & 3,5 \\
\hline $\begin{array}{l}\text { Relacionamento } \\
\mathrm{c} / \text { chefias }\end{array}$ & 4,0 & 4,5 & 4,0 & 4,5 \\
\hline $\begin{array}{l}\text { Relacionamento } \\
\text { c/ outros profis. }\end{array}$ & 4,3 & 4,4 & 4,3 & 4,4 \\
\hline $\begin{array}{l}\text { Liberdade p/ } \\
\text { decisões }\end{array}$ & 3,0 & 3,0 & 3,5 & 3,5 \\
\hline
\end{tabular}




\section{continuação da Tabela 5}

Tabela 5 Escore médio estimado das avaliações de condições de trabalho, segundo itens pesquisados entre absenteístas e não absenteístas dos Hospitais A e B, São Paulo, 2001.

\begin{tabular}{|c|c|c|c|c|}
\hline \multirow{2}{*}{ Item } & \multicolumn{2}{|c|}{ Absenteísta } & \multicolumn{2}{|c|}{ Não absenteísta } \\
\hline & $\underset{n=87}{A}$ & $\begin{array}{c}B \\
n=99\end{array}$ & $\begin{array}{c}A \\
n=25\end{array}$ & $\mathrm{~B}=41$ \\
\hline $\begin{array}{l}\text { Reconhecimento da } \\
\text { empresa p/ esforços }\end{array}$ & 2,6 & 3,1 & 3,1 & 3,6 \\
\hline $\begin{array}{l}\text { Treinamento e } \\
\text { des. atividades }\end{array}$ & 3,9 & 4,4 & 3,9 & 4,4 \\
\hline $\begin{array}{l}\text { Perspectiva de } \\
\text { desenv. profis. }\end{array}$ & 3,0 & 3,2 & 3,4 & 3,7 \\
\hline $\begin{array}{l}\text { Pausas informais } \\
\text { no trabalho }\end{array}$ & 3,2 & 4,0 & 3,2 & 4,0 \\
\hline Escala trabalho & 3,2 & 3,9 & 3,9 & 3,9 \\
\hline Assist. médica & 2,0 & 4,5 & 2,8 & 4,5 \\
\hline Satisfação trabalho & 3,9 & 4,4 & 4,2 & 4,6 \\
\hline
\end{tabular}

Quanto à avaliação das condições de trabalho pelos trabalhadores (TABELA 5), aplicando o escore de 1 a 5 , sendo 1 indicativo da pior condição e 5 da melhor e considerando o ponto médio 3 , dos 21 itens constantes no questionário a maioria obteve escore maior que 3. No hospital A, entre absenteístas e não absenteístas os itens que obtiveram escore menor que 3 foram: calor, espaço de trabalho e assistência médica. Já no hospital B somente o item calor obteve escore menor que 3 tanto entre absenteístas quanto não absenteístas.

Dentre os itens que obtiveram escore maior que 3, pela análise estatística a um alfa $=20 \%$, o efeito de hospital foi significativo nos itens: iluminação, espaço de trabaIho, relacionamento com colegas, com chefias e com outros profissionais, treinamento $e$ desenvolvimento de atividades e pausas informais no trabalho, ou seja, o escore médio foi maior tanto entre absenteístas quanto não absenteístas do hospital $B$ em relação ao $A$.

\section{Discussão}

As razões estimadas, pelo modelo estatístico, da taxa de freqüência por episódios e da proporção de tempo perdido não demonstraram que em relação ao absenteísmo dos trabalhadores dos SNDs, o hospital público apresente situação pior que o hospital privado.

O interessante foi observar que, no hospital público, os trabalhadores apresentaram absenteísmo superior que as trabalhadoras, contrariando os resultados obtidos por NOGUEIRA (1980), NIETO et al. (1987) e RITCHIE (1999). Entretanto, pode-se considerar esse resultado atípico, pois um trabalhador do hospital público teve o episódio mais longo, com 212 dias de duração por licença I.N.S.S., fato que não ocorreu entre as trabalhadoras de ambos os hospitais, nem entre os trabalhadores do hospital privado. $\bigcirc$ fato de as mulheres apresentarem absenteísmo mais elevado que os homens é um dado agra- 
vante para o Serviço de Nutrição e Dietética, cuja população é normalmente constituída por mulheres. Em nosso estudo, a população feminina representou $86 \%$ no Hospital A e $67 \%$ no Hospital B. A diferença no absenteísmo entre homens e mulheres, segundo MASTEKAASA et al. (1998), não decorre das condições de trabalho nem da maior responsabilidade imposta às mulheres pelo cuidado a filhos, mas, sim, das condições gerais de saúde e das diferenças de personalidade. Os efeitos das diferentes fontes de estresse na vida e no trabalho sobre o bem estar físico e emocional são diferentes para homens e mulheres. Segundo HENDRIX (1994), a variável sexo modera o efeito dessas fontes de estresse, as mulheres sofrem níveis mais elevados de estresse no trabalho e de absenteísmo e baixos estados emocionais de bem estar.

Em relação à faixa etária, ao tempo de trabalho no serviço e ao cargo, confirmando dados da literatura, o absenteísmo foi mais elevado, entre os mais jovens, entre os trabalhadores com menos tempo de trabalho e entre os trabalhadores operacionais.

Também, confirmando dados da literatura estudada, os trabalhadores com responsabilidade no cuidado a crianças (filhos, netos ou sobrinhos) tiveram absenteísmo mais elevado que aqueles sem essa mesma responsabilidade, tanto no hospital público quanto no privado. Destacou-se, com base na análise estatística, a interação entre responsabilidade com crianças e gênero. As mulheres que tinham essa responsabilidade obtiveram valores mais elevados que os homens com a mesma responsabilidade, diferença não observada entre homens e mulheres que não tinham essa responsabilidade. As demandas familiares estão associadas a níveis elevados de burnout e, conseqüentemente, de ausências no trabalho. Trabalhadores que têm sob suas responsabilidades crianças menores de 6 anos ou que têm dificuldades no cuidado aos filhos são os que estão sujeitos a esse risco e necessitam de políticas de suporte familiar (ERICKSON et al, 2000). Essa questão merece atenção especial das chefias e da equipe de Medicina do Trabalho para o controle do absenteísmo nas empresas, pois observa-se que as mulheres estão assumindo a responsabilidade pelo cuidado a filhos e netos, neste caso devido à gravidez das filhas adolescentes, em idade cada vez mais jovem.
Em nosso estudo, os trabalhadores com responsabilidade no cuidado a crianças representaram cerca de $50 \%$ da população estudada.

Apesar de os episódios de ausências por doença própria, de curta duração, terem sido mais freqüentes em ambos os hospitais, em relação ao número de dias de trabalho perdidos, os episódios de doenças com duração de 4 a 15 dias foram os maiores responsáveis no hospital público (332 dias), seguidos de licenças I.N.S.S. (247 dias). No hospital privado, as licenças I.N.S.S. representaram o maior número de dias de trabalho perdidos (713 dias) e foram seguidas de episódios com duração de 4 a 15 dias $(631$ dias). Essas ausências com duração de até 15 dias constituem problema de muita importância, pois não há substituição do funcionário, o que implica em acúmulo de trabalho para os demais trabalhadores, redução de suas folgas de direito no mês e, conseqüentemente, acúmulo de cansaço físico e mental que podem gerar estresse ocupacional e problemas de saúde como as doenças cardiovasculares, distúrbios gastrointestinais, distúrbios de sono e depressão, citados por PARAGUAY (1990). Esses funcionários, provavelmente, serão os futuros absenteístas, apontados nos estudos realizados por MCKEVITT et al. (1997) e GRINYER; SINGLETON (2000), tornando o círculo vicioso.

Os acidentes de trabalho, neste estudo, a quarta e terceira causas de episódios de ausências, com perdas de 322 dias e de 124 dias de trabalho, correspondendo a $19 \%$ e a $5 \%$, respectivamente, do total de dias de ausências nos hospitais público e privado, são muito estudados nos Serviços de Nutrição e Dietética (MORRONE;GARCIA, 1978 e VALTORTA, 1985) por fazerem parte de um ambiente hospitalar, que é mais propício aos acidentes pelas próprias características e condições de trabalho em que os funcionários são submetidos, como por exemplo: altas temperaturas; pisos escorregadios; carregamento de pesos; ritmo intenso de trabalho; manuseio de equipamentos elétricos, a vapor e a gás e de utensílios cortantes; além de aspectos de organização do trabalho que podem levar ao estresse ocupacional e, conseqüentemente, aos acidentes de trabalho. É exigido dos trabalhadores dos Serviços de Alimentação uma alta produtividade em tempo limitado, em condições muitas vezes inadequadas 
de trabalho, por falta de um planejamento físico correto, com cruzamentos de fluxos e espaços reduzidos em relação ao número produzido de refeições. As condições inadequadas geram insatisfações, queda de produtividade, problemas de saúde e acidentes de trabalho. Em nosso estudo, embora os acidentes tenham ocorrido em maior proporção no próprio serviço, destacaram-se os acidentes de trajeto, da casa para o trabalho no hospital público e, no sentido inverso, no hospital privado. Sabe-se que a maioria dos trabalhadores, principalmente operacionais, residem longe dos locais de trabalho, que os obrigam a utilizar vários meios de transporte; que muitos saem de suas casas sem se alimentarem adequadamente e aqueles que têm sob seus cuidados crianças pequenas são obrigados a levá-las e a buscá-las nas creches ou escolas, além do que a maioria das trabalhadoras são submetidas a tarefas domésticas quando retornam para suas residências. Hoje, muitas mulheres estão assumindo o papel de chefe de família, o que as obriga a buscarem trabalhos extras, como realização de faxina em residências, em seus dias de folgas, para complementarem o salário. Todos esses fatores contribuem no aumento de riscos de acidentes de trabalho pelo acúmulo de cansaço e de responsabilidade. Se, durante a jornada diária de trabalho, não forem observadas pausas, o risco pode aumentar, o que pode justificar, neste estudo, o número maior de acidentes no primeiro turno de trabalho, ou seja, de até 4 horas após seu início. Esse assunto também merece um estudo específico e ações dos profissionais da Medicina do Trabalho e dos Serviços, para conscientização dos trabalhadores quanto aos riscos de acidentes e à importância do uso de equipamentos de proteção individual (EPI), para melhoria das condições de trabalho e para implantação de programas de proteção ao trabalhador.

É indiscutível o prejuízo das ausências dos trabalhadores no desempenho dos hospitais, sejam públicos ou privados, mais do que de outras organizações, pelas características já apontadas. Em nosso estudo, como era de se esperar, foi unânime a opinião dos servidores quanto ao prejuízo provocado pelo absenteísmo de membros da equipe no desempenho das funções no SND, tanto em hospitais públicos quanto privados.
Nas sugestões apresentadas pelos trabalhadores para diminuição das ausências ao trabalho, destacaram-se a necessidade dos mesmos de serem ouvidos pelas chefias e a valorização em relação ao papel das chefias na gestão de pessoas, inferindo pela preferência às chefias mais rigorosas, mas justas e imparciais. Torna-se imprescindível, portanto, o treinamento e desenvolvimento dos profissionais que ocupam cargos de direção e que têm sob seu comando trabalhadores de diversos níveis de escolaridade, na gestão de pessoas e na aquisição de conhecimentos na área de saúde do trabalhador. Esses resultados vêm ao encontro de estudo realizado por HOVERSTAD; KJOLSTAD (1991) com grupos focais de trabalhadores, de indústria e de companhia de seguro, sobre os motivos atribuídos pelos mesmos para o absenteísmodoença. O papel da chefia foi citado como terceira causa, entre as condições de trabatho, que influencia o absenteísmo, precedida pela sensação de bem estar, entendida como relações sociais existentes no local, e pela organização do trabalho. A questão do relacionamento da equipe de trabalho é apontada como mais importante que outros fatores psicossociais como carga e autonomia no trabalho, no absenteísmo-doença, também entre os profissionais da área de saúde, segundo estudo de KIMIVÄKI et al (2001).

A resposta "melhorar as condições de trabalho", com maior percentual entre os trabalhadores do hospital público, justifica-se pela necessidade de reforma e de ampliação da área física da cozinha, decorrente do aumento progressivo do número de leitos hospitalares, com conseqüente aumento na produção de refeições, no número de equipamentos e no aumento de servidores na área. Essa necessidade de reforma na área física também explica os escores mais baixos atribuídos aos itens espaço de trabalho, calor e ruído, pelos trabalhadores do hospital público. Destacou-se, também no hospital público, o escore mais baixo observado na questão assistência médica, tanto entre absenteístas quanto não absenteístas, considerado um dos fatores de maior insatisfação entre os trabalhadores desse hospital.

A boa interação existente entre as pessoas e a atenção ao treinamento e desenvolvimento oferecido, em ambos os hospitais, foram consideradas, pelos trabalhadores, como fa- 
tores positivos. Consideramos esses quesitos de fundamental importância, pois podem contribuir como facilitadores para implantação de ações, comentadas ao longo do texto, na redução da situação do absenteísmo existente nos dois hospitais.

Pelo visto, os motivos atribuídos ao absenteísmo de trabalhadores dos SNDs são semelhantes nos hospitais públicos e privados e a outras organizações não hospitalares. Pela própria característica desses serviços e por serem serviços que ocupam lugar de destaque na assistência direta prestada ao paciente, a questão do absenteísmo é preocupante, pois traz sobrecarga de trabaTho a aqueles que são assíduos, gera insatisfação no grupo, prejudica o desenvolvimento e a qualidade do trabalho prestado ao paciente, comprometendo, consequentemente, a eficácia organizacional.

Tornam-se necessárias, portanto, além de reestudos específicos com trabalhadores de SNDs de outros hospitais brasileiros, ações efetivas e integradas por parte:

- das chefias na promoção da satisfação pelo trabalho e no suporte social;

- do Serviço de Medicina do Trabalho nas avaliações, controles e divulgações dos absenteísmos por doenças e por acidentes de trabalho; nas determinações e orientações das condições de trabalho e na promoção da saúde do trabalhador e

- da empresa por meio de políticas, no reconhecimento dos esforços dos trabalhadores e na melhoria da qualidade de vida no trabalho.

\section{Referências bibliográficas}

ERICKSON,RJ; NICHOLS,L; RITTER,C. Family influences on absenteeism: testing an expanded process model. Journal of Vocational Behavior; 57(2): 246272, 2000.

\section{FISCHER,FM. Condições de trabalho e de vida em trabalhadores de se- tor petroquímico [Tese de Livre- Docência].São Paulo: Faculdade de Saú- de Pública da Universidade de São Pau- lo;1990.}

GRINYER,A; SINGLETON,V. Sickness absence as risk-taking behaviour: a study of organizational and cultural factors in public sector. Health, Risk \& Society; 2:7-21, 2000.

HENDRIX,WH;SPENCER,BA;GIBSON,GS. Organizational and extraorganizational factors affecting stress, employee wellbeing, and absenteeism for males and females. Journal of Business and Psychology; 9(2):103-128, 1994.
HOVERSTAD,T; KJOLSTAD,S. Use of focus groups to study absenteeism due to illness. Journal of Occupational Medicine; 33(10):1046-1050, 1991.

Intercooled Stata 7.0. Statistics/Data Analysis. Stata Corporation. College Station, Texas, 1984-2002.

KIVIMÄKI,M; SUTINEN,R; ELOVAINIO,M; VAHTERA,J; RÄSÄNEN,K; TÖYRY, S et al. Sickness absence in hospital phsysicians: 2 year follow up study on determinants. Occup Environ Med; 58:361-366, 2001.

MASTEKAASA,A; OLSEN,KM. Gender, absenteeism, and job characteristics - a fixed effects approach. Work and Occupations; 25(2):195-228, 1998.

MCKEVITT,C; MORGAN,M; DUNDAS,R; HOLLAND,WW. Sickness absence and working through illness: a comparison of two professional groups. Journal of 
Public Health Medicine; 19:295300, 1997.

MONIZ,AE. Controle estatístico do ausentismo-doença. Saúde Ocup Seg. ; XIII (1):26-33, 1978

MORRONE,LC; GARCIA,M. Acidentes de trabalho em um hospital. In: Anais do XVII Congresso Nacional de Prevenção de Acidentes do Trabalho; out 1-4; São Paulo, 1978.

NIETO,ZO; TAPIAS,BH; ORTIZ,AC; MATURANA,M. Ausentismo laboral por enfermedad Hospital Regional de Caldas Antioqua 1985. Bol Epidemiol Antioq ; 12(2):169-83, 1987.

NOGUEIRA,DP. Absenteismo-Doença: Aspectos epidemiológicos [Tese de Livre-Docência]. São Paulo: Faculdade de Saúde Pública da Universidade de São Paulo;1980.
PARAGUAY,AIBB. Estresse, conteúdo e organização do trabalho: contribuições da ergonomia para melhoria das condições de trabalho. Rev Bras de Saúde Ocupacional ; 18(70):40-43, 1990.

RITCHIE, KA; MACDONALD, EB; GILMOUR,WH; MURRAY,KJ. Analysis of sickness absence among employees of four NHS trusts. Occup Environ Med; 56:702-708, 1999.

SAS System 8.2 Business Intelligence and Analytics. SAS Institute Inc.Cary, NC, 1999-2001.

S-PLUS 6.0 Professional 2. Statistical Analysis. Insightful Corp. Seattle, WA, 1988-2001.

VALTORTA,A; SIDI,E; BIANCHI,SCL. Estudo do absenteismo médico num hospital de grande porte. Rev Bras Saúde Ocupacional; 13:55-61, 1985. 